\title{
La acción social empresarial como instrumento de Justicia Social: la empresa como garante de los Derechos Humanos
}

\section{Corporate Social Action as an Instrument of Social Justice: the Company as Guarantor of Human Rights}

\section{CARMEN MARÍA GÓMEZ NAVARRO \\ Departamento de Trabajo social y Servicios Sociales. Universidad de Murcia \\ carmen.gomeznavarro@um.es}

Resumen: La Justicia Social y los Derechos Sociales son la filosofía de valores presentes en el modelo social internacional y la Responsabilidad social corporativa (RSC) que ofrece una alternativa al enfoque de protección de los Derechos Humanos desde la perspectiva de las cuestiones socioeconómicas Suponen un avance conforme a la filantropía o el marketing social y nos permite incluir en sus metodologías y acciones el modelo de la Base de la Pirámide (BdP).

A lo largo del presente trabajo se recoge la vinculación entre la RSC, los negocios inclusivos y los de Base de la Pirámide en la lucha contra la exclusión social y como forma de trabajo para garantizar los Derechos Humanos en el mundo empresarial y el papel que tiene el trabajador social en el mundo empresarial como agente de desarrollo de la Responsabilidad Social Corporativa tanto a nivel interno como externo.

Palabras clave: Negocios Inclusivos*, Política Social, Responsabilidad Social de la Empresa, Políticas sobre Derechos Humanos, Ética Social.

\section{SERGIO FERNÁNDEZ RIQUELME}

Departamento de Trabajo social y Servicios Sociales. Universidad de Murcia

serferi@um.es

Abstract: Social Justice and social rights are the philosophy of values present in the international social model and the social responsibility corporate (CSR) that offers an alternative to the approach for the protection of human rights from the perspective of the socioeconomic issues, supposing an advance pursuant to philanthropy or social marketing and allows us to include in its methodologies and actions the Base of the pyramid (BoP).

Along the present work includes linking CSR, inclusive businesses and those of BdP in the fight against social exclusion and as a form of work to secure human rights in the business world and the role of the social worker in the business world as an agent of development of CSR has both internally and externally.

Key Words: Inclusive Business, Social Policy, Corporate Social Responsibility, Human Rights Policy, Social Ethics 


\section{INTRODUCCIÓN}

La paz universal y duradera sólo puede alcanzarse si se basa en la Justicia Social, respondiendo a las necesidades de los individuos y de la comunidad a través del esfuerzo conjunto de Estado, Empresa y Sociedad civil.

Los actuales índices de pobreza sitúan a más de 1000 millones de personas viviendo en la pobreza a nivel mundial ${ }^{1}$ lo que genera desigualdades socioeconómicas, característica definitoria del modelo de desarrollo presente en buena parte de nuestras sociedades actuales, como se aprecia en la Figura 1. No todos los ciudadanos tienen acceso ni disfrutan de las ventajas que supone un Estado de Bienestar; no tienen cubiertas las necesidades básicas, materiales y no materiales de la persona.

\section{Figura 1}

\section{Población bajo el nivel de pobreza}
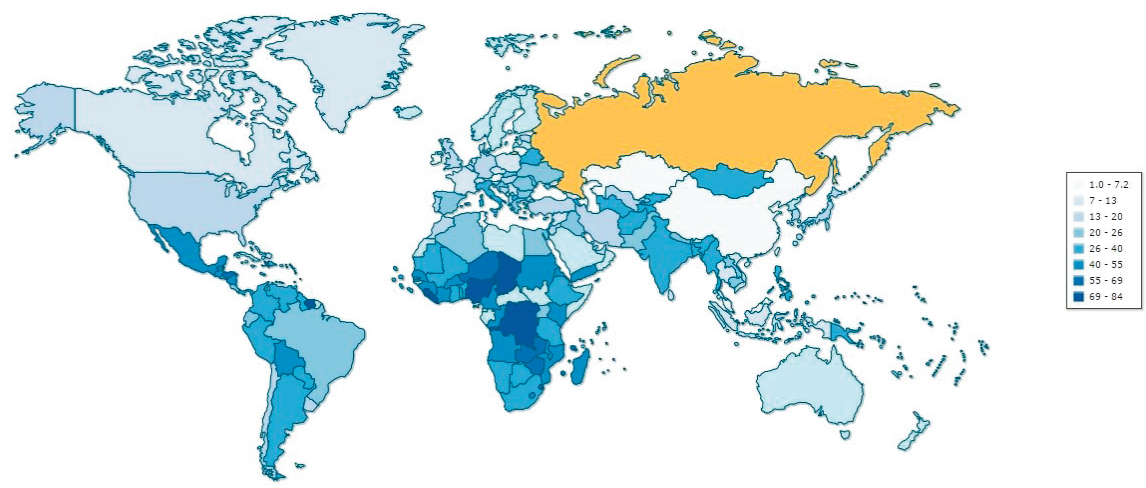

Fuente: CAI World Factbook, (2014).

Para alcanzar el pleno cumplimiento de los Derechos Humanos, así como para realizar cambios en los estilos de vida que nos permitan avanzar hacia sociedades democráticas y equitativas debemos mirar con otros ojos el mundo empresarial. Debemos ser capaces de tener una visión que nos permita luchar

1 World Bank (2016). 
por los principios de solidaridad, cooperación, creatividad, innovación, trabajo en equipo, reflexión crítica y análisis de la realidad, participación o movilización activa por el bien común.

En esta línea se aprobaron los Objetivos de Desarrollo Sostenible (ODS) marcados como compromiso de los 191 gobiernos y estados presentes en las Naciones Unidas para luchar contra la pobreza extrema en sus diferentes dimensiones: hambre, salud, educación de calidad, agua, clima, etc.

Nuestra estrategia ante la empresa debe ser la de su conversión en un motor de cambio individual, grupal y comunitario. Frente al problema de la pobreza, de la desigualdad social, económica y política se debe apostar por una estrategia de inclusión de las empresas, así como de las políticas de mercado y de empleo para generar valor social y favorecer la inclusión.

De hecho, desde 2011, nos recuerdan Bonguetxea y Millán (2016), en España existe una ley en la que se reconoce la especificidad de un modelo de empresa cuya finalidad es la satisfacción de las necesidades sociales. Hablamos de la ley 5/2011 de Economía Social ${ }^{2}$

La consideración del sector empresarial dentro del marco de los Derechos Humanos no es un invento nuevo ni una salida desesperada a la situación de crisis económica y social que se vive desde hace años, sino que es una de las bases que sustentó el nacimiento de las Naciones Unidas, organismo creado para garantizar la paz universal y el desarrollo de los pueblos.

La Organización de las Naciones Unidas (ONU), en su carta inaugural de $1945^{3}$, recoge los derechos civiles y políticos (artículos 3 a 21) y los derechos económicos, sociales y culturales (artículos 22 a 27) constituyendo la Declaración Universal de Derechos del Hombre (DUDH) $(1948)^{4}$ que posteriormente serían desarrollados en el Pacto Internacional de los Derechos Civiles y Políticos ${ }^{5}$ y el Pacto Internacional por los Derechos Económicos, Sociales y Culturales ${ }^{6}$ (ambos aprobados por la Resolución 2200 A (XXI), de 16 de diciembre de 1966). La relación entre el desarrollo normativo en materia de DDHH y su vinculación con la empresa y la justicia social se puede apreciar

2 Ley 5/2011, de 29 de marzo, de Economía Social. Publicada en el BOE, núm. 76, de 30 de marzo de 2011.

3 http://www.un.org/es/charter-united-nations/.

4 https://documents-dds.ny.un.org/doc/RESOLUTION/GEN/NR0/046/82/IMG/NR004682.pdf?OpenElement.

5 http://www.ohchr.org/SP/ProfessionalInterest/Pages/CCPR.aspx.

6 http://www.ohchr.org/SP/ProfessionalInterest/Pages/CESCR.aspx. 
en la Figura 2. De esta manera, se observa que en 2011, el Consejo de Derechos Humanos de la ONU adoptó por consenso los Principios Rectores sobre empresas y Derechos Humanos (A/HRC/17/31), quedando así consagrados como la norma, jurídicamente no vinculante, de conducta a nivel mundial que se espera de todas las empresas y de todos los Estados en relación con las empresas y los Derechos Humanos (DDHH), y recogiendo la "obligación del Estado de proteger a las personas de potenciales abusos cometidos por empresas y la responsabilidad de la empresa de respetar los Derechos Humanos, y la obligación del Estado de garantizar la reparación de las personas afectadas por violaciones de sus derechos"7.

\section{Figura 2}

Línea temporal del desarrollo normativo en materia de Derechos Humanos, Justicia Social y Empresa

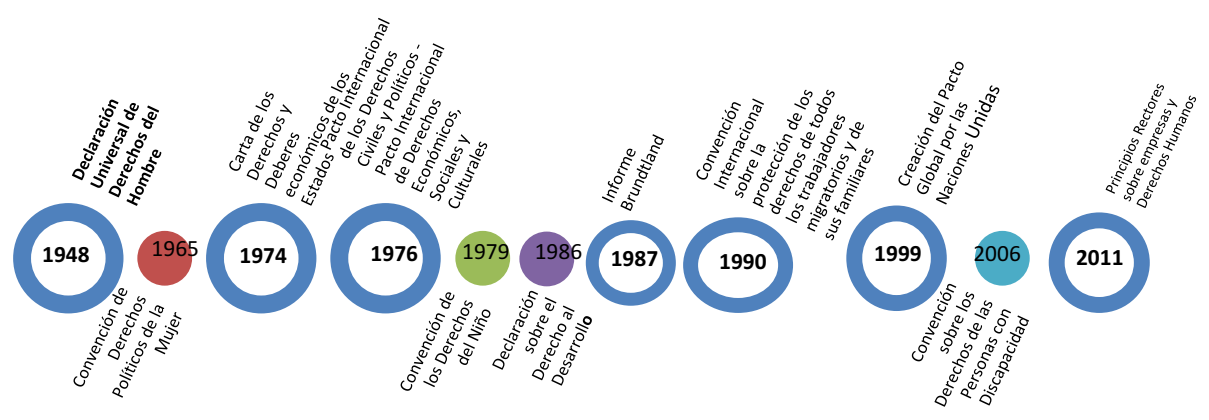

Fuente: Elaboración propia.

La Justicia Social y los Derechos Sociales son la filosofía de valores presentes en el modelo social internacional, y se constituyen como un marco ideológico desde el cual entender un espacio de trabajo y gestión de la ética profesional, laboral y empresarial. A esta filosofía se le suma la Responsabilidad Social Corporativa (RSC) ofreciendo una alternativa al enfoque de protección de los Derechos Humanos desde la perspectiva de las cuestiones socioeconómicas.

7 Márquez Carrasco, C. (2014), p. 7. 
La globalización de la economía tiene sus luces y sus sombras. A la vez que ha supuesto oportunidades y beneficios para muchas personas, también ha conllevado el desplazamiento de trabajadores y empresas a otros emplazamientos. Provoca cambios en la forma de entender lo económico, ambiental y social tanto de la comunidad receptora como de la comunidad que perdía a las empresas y sus trabajos, ocasionando, en positivo y en negativo, rápidos y severos cambios de capital e inestabilidad financiera.

La Organización Internacional del Trabajo sostiene que "las reglas que rigen la economía global deberían destinarse a mejorar los derechos, los medios de subsistencia, la seguridad y las oportunidades para las personas, las familias y las comunidades en todo el mundo" "y por ello, el rol jugado por el sector privado, y más concretamente por la empresa y las corporaciones supra internacionales, para combatir la pobreza y las desigualdades, ha sido sujeto de un gran debate internacional, tanto en espacios académicos como no académicos ${ }^{9}$.

En este escenario ha habido un innegable avance en la forma de comprender y de implantar la Responsabilidad Social Corporativa (RSC) lo que supuso el avanzar, en no pocas ocasiones, más allá de la filantropía o del marketing social y nos permite incluir en sus metodologías y acciones la Base de la Pirámide (BdP).

\section{CONSIDERACIONES PREVIAS}

\section{Fusticia Social, Derechos Humanos, Ética y Empresa}

Los Derechos Humanos son inherentes a nuestra naturaleza y, junto a las libertades fundamentales, nos permiten desarrollar y emplear cabalmente nuestras cualidades humanas, nuestra inteligencia, nuestro talento y nuestra conciencia así como satisfacer nuestras variadas necesidades, entre ellas las necesidades espirituales ${ }^{10}$.

Las libertades básicas y los derechos fundamentales recogidos en la Declaración Universal de los Derechos Humanos (DUDH) constituyen los

\footnotetext{
8 Comisión Mundial sobre la Dimensión Social de la Globalización (2004), p. 159.

9 Frynas, J. G. (2005); Frynas, J. G. (2008); Jamali, D. J. (2010); Lodge, G. y Wilson, C. (2006); Muthuri, J. N.; Moon, J. e Idemudia, U. (2012); Van Sandt, C. W. y Sud, M. (2012).

10 Naciones Unidas (1987).
} 
principios básicos de Justicia Social de las Naciones Partes que configuran la ONU. El deseo de alcanzar una sociedad igualitaria, libre y justa es lo que mueve a los pueblos a crear un marco jurídico que recoja los derechos y obligaciones de Estado, sociedad y empresa para garantizar el cumplimiento y defensa de los bienes superiores recogidos en la citada Declaración.

Pero todo derecho lleva, de manera correlativa, una obligación, al igual que todo deber tiene su derecho, recoge Moix ${ }^{11}$ y tal es la fuente de los derechos sociales y de la Justicia Social que consiste en establecer en cada sociedad el equilibrio de los derechos y de los deberes. La Justicia Social se sustenta sobre los principios de justicia distributiva y de justicia conmutativa, y debido a ello se considera este sistema político como responsable de la "procura existencial".

La autoridad política que debe buscar la Justicia Social surge del consentimiento entre individuos libres de depositar su libertad inicial en una autoridad comunitaria. De esta forma, sin negar la existencia de un derecho natural, los orígenes de la norma y del derecho surgen de poner los derechos colectivos y la búsqueda del bien común por encima de la búsqueda exclusiva del interés particular. Los ciudadanos ceden su poder a la comunidad civil creando así las leyes, que deben asegurar la Justicia Social, a través de su elección del poder civil o de la autoridad de gobierno que han decidido formar.

Si seguimos el sistema teórico de Justicia Social promulgado por Rawls (1995), éste otorga prioridad absoluta a la justicia y la entiende como "la primera virtud de las instituciones sociales" (familia, ley, constitución política, protección jurídica de libre pensamiento y de la propiedad privada de los medios de producción, entre otros). Así, la Justicia Social es la estructura básica de la sociedad y, especialmente, el modo en que las instituciones sociales distribuyen deberes y derechos en la sociedad. De esta forma se puede realizar una repartición adecuada de los beneficios y de las cargas que supone la cooperación social, que prima sobre la búsqueda del interés personal en caso de conflicto de intereses, y que se basa en la necesidad de trabajar conjuntamente para superar las limitaciones de los recursos y alcanzar un mayor bien para todos.

Esta idea es la base de la propia constitución de la ONU, pues se recoge expresamente en el art. 1 de la Carta de las Naciones Unidas ${ }^{12}$ el principio de

11 Moix, M. (1977).

12 http://www.un.org/es/sections/un-charter/chapter-i/index.html 
cooperación social para garantizar la Justicia y para la "solución de problemas internacionales de carácter económico, social, cultural o humanitario, y en el desarrollo y estímulo del respeto a los Derechos Humanos y a las libertades fundamentales de todos".

Por eso "una sociedad está bien ordenada no sólo cuando fue organizada para promover el bien de sus miembros, sino cuando también está eficazmente regulada por una concepción pública de la justicia" ${ }^{13}$. Entonces, los Derechos Humanos implican obligaciones pero ¿en quién recae la obligación de realizarlos? Onora ONeill argumenta, por ejemplo, que el Estado tiene el papel fundamental de la Justicia Social mientras que otros agentes, como las empresas o el Tercer Sector ${ }^{14}$, pueden contribuir de manera relevante no sólo atendiendo su deber negativo de respetar los Derechos Humanos, sino también con su deber positivo de protegerlos y promoverlos ${ }^{15}$. El Derecho Internacional debe crear los mecanismos de responsabilidad necesarios y vinculantes para los Estados de forma que los Derechos Humanos sean de obligado cumplimiento y sean parte integral del enfoque de derechos positivos internacionales.

Esto se traduce en que se debe establecer una línea de asignación de entes con deberes positivos (es decir, proteger, promover o realizar) hacia los Derechos Humanos, directamente obligados a cumplir y a no socavar la asignación de tareas según la distribución con base en la Justicia Social. Entendemos que es el Estado quien tiene el deber primordial de implantarlos y sería, pues, el primero en esa línea de sucesión de obligaciones.

Asimismo, la carta de la ONU reconoce como uno de sus principios el de cooperación pacífica entre los Estados y el respeto a los Derechos Humanos, por lo que en el caso de que un Estado no tuviera capacidad de ofrecer la protección a estos Derechos, será la Comunidad Internacional quien acuda, en un deber secundario, para hacer frente a esta tarea, obligación que vuelve a copiar el Comité del Pacto Internacional de Derechos Económicos, Sociales y Culturales, en su Observación General n ${ }^{\circ}$ 3, estableciendo la obligación de todos los estados de garantizar estos derechos y, en el caso de que el único

13 Rawls, J. (1995), p. 18.

14 Por descarte, si el primer sector es el Estado y el segundo es el mercado, el tercer sector está formado por organizaciones sin ánimo de lucro, asociaciones y fundaciones, y por cooperativas. O’Neill, O. (2001), pp. 181-182.

15 Kolstadt, I. (2009). 
obstáculo para ello fuera la falta de recursos económicos, ofrece como recurso el acudir a la cooperación internacional para el desarrollo.

Supondría, pues, que en un Estado Social, democrático y desarrollado, las empresas sólo tendrán funciones limitadas en el sentido negativo, mientras que en los países en desarrollo o con regímenes autoritarios son las empresas las que, en un sentido práctico, atienden estas necesidades, si bien se mantiene vigente el principio de cooperación para otros Estados o para la Comunidad Internacional.

Por otro lado hay que esgrimir el argumento crítico de que un derecho sólo es plausible si el deber correlativo es igualmente plausible, así el deber de abastecer las necesidades básicas de los seres humanos en estado de necesidad no es plausible. Las implicaciones del enfoque de las capacidades incluyen un concepto amplio de los Derechos Humanos que tenga en cuenta la pobreza mundial, el rechazo al absolutismo y que el obstáculo teórico de la limitación de recursos representa un obstáculo teórico a las obligaciones legales internacionales de Derechos Humanos ${ }^{16}$. Por ello, la ONU establece que los programas de desarrollo deben promover los principios internacionales de Derechos Humanos y reforzar la capacidad de los "titulares de deberes" para cumplir con sus obligaciones de DDHH y promover la capacidad de los "titulares de derechos" para reclamar sus Derechos Humanos ${ }^{17}$. Ante el discurso de que en los Derechos Humanos no son oponibles Estado y actores no estatales, podemos argumentar que la distinción entre la esfera pública y privada como lugares apropiados e inapropiados para la aplicación de Derechos Humanos respectivamente, es articial, contraproducente y opresiva.

El marco de la $\mathrm{ONU}^{18}$ reconoce el deber de los Estados de proteger los Derechos Humanos que pueda satisfacerse mediante informes de sostenibilidad y que estos deben relacionarse con la diligencia debida de las corporaciones, término que se refiere al estándar para determinar la existencia de responsabilidad internacional del Estado, en particular, en relación a las actividades de actores privados ${ }^{19}$. En sus Principios Rectores de Naciones Unidas sobre Empresas y Derechos Humanos ${ }^{20}$, aprobados en 2011, el Representante Especial del Secretario General (RESG) continúa esta labor, definiendo específicamente qué significan y cómo se han de desarrollar los

\footnotetext{
16 Osuji, O. K. (2014).

17 Ghai, Y. y Cottrel, J. (2004), pp. 58-60.

18 Naciones Unidas (2008).

19 Martín Ortega, O. (2013), p. 168.

20 Naciones Unidas (2011).
} 
conceptos propuestos en el marco: la obligación del Estado de proteger, la responsabilidad de las empresas de respetar y la necesidad de remediar los ataques o las agresiones que contra ellos se produzcan.

\section{2. Ética empresarial: los Derechos Humanos como valor supremo de la empresa}

La actual situación social y económica mundial ha facilitado una visión de las multinacionales y las grandes empresas como responsables de la crisis económica, por lo que estas se ven en la necesidad, casi en la obligación, de marcar de manera clara y contundente su misión, los valores que defienden y la estrategia que siguen para alcanzar a corto y a medio plazo sus objetivos.

Es decir, las organizaciones empresariales se definen y se presentan ante la sociedad por cómo hacen su trabajo: cómo es su cadena de producción, cuál es su relación con los distribuidores y los proveedores, y quién es su mercado potencial. Tratar de equilibrar estos elementos con las pretensiones de una sociedad socialmente más exigente y ambientalmente concienciada dará lugar a su "cadena de valor".

$\mathrm{Al}$ igual que la Responsabilidad Social Empresarial (RSE), el concepto de desarrollo sostenible continúa siendo debatido, refutado y reinterpretado en la literatura nacional e internacional. Cualquier iniciativa de Responsabilidad Social Corporativa debe ser sensible al contexto y tomar en cuenta posibles diferencias económicas, sociales, políticas, ambientales, culturales y organizacionales ${ }^{21}$.

Los objetivos que pueden llevar a las empresas a dar participación a sus grupos de interés son morales o estratégicos ${ }^{22}$. La ética empresarial debe partir de que, ya se hable de RSC, de sostenibilidad empresarial o de negocio inclusivo, ésta supone integrar a los stakeholders en los procesos de decisión, desarrollando tanto la Responsabilidad Social corporativa interna, mejorando el ambiente de trabajo, como la Responsabilidad Social corporativa externa, creando cadenas de valor.

En la erradicación de la exclusión social, afirman Castel (2004), Moriña (2007) y Tezanos $(2008)^{23}$, destacan cuatro grandes grupos de agente: a) Las ONG y los servicios sociales b) Agentes privados, entre ellos las empresas c) Las instituciones públicas d) Los gobernantes. En el contexto empresarial

21 Barkemeyer, R. (2011).

22 Noland, J. y Phillips, R. (2010).

23 Castel, R. (2004); Moriña, A. (2007) y Tezanos, J. F. (2008). 
recientemente han adquirido gran importancia conceptos como empresas sociales, negocios inclusivos o RSC, ideas que se desarrollan a lo largo de este documento.

3. Nuevas formas de hacer negocio para abordar la exclusión: RSC, Negocio inclusivo y Base de la Pirámide

El fenómeno de la exclusión social se suele relacionar con la pobreza, pero es un concepto multifactorial que abarca aspectos más allá de los ingresos económicos, sus dimensiones abarcan lo laboral, lo económico, lo cultural, lo personal y lo socio-relacional ${ }^{24}$. Por tanto, el proceso de trabajo para prevenir y superar la exclusión social debe abordarse desde distintas actuaciones, trabajando con la familia, la escuela, y también con la empresa.

El Libro Verde sobre RSC de la Unión Europea ${ }^{25}$ la define como la integración voluntaria, por parte de las empresas, de las preocupaciones sociales y medioambientales en sus operaciones comerciales y en sus relaciones con sus interlocutores. Estos interlocutores, grupos de interés o stakeholders pueden ser tanto externos (consumidores, proveedores...), como internos (empleados y directivos).

En respuesta a las ideas equivocadas acerca de la Responsabilidad Social Corporativa que la equiparan a la filantropía o al marketing social, varios autores hemos argumentado a favor de una evolución conceptual que nos lleve a una interpretación más holística de la noción de RSC, Responsabilidad Social Corporativa 2.0. La Responsabilidad Social Corporativa 2.0 implica una gestión empresarial holística enfocada a la sostenibilidad, respondiendo a todas las cuestiones sociales, económicas y ambientales.

De esta manera, la Responsabilidad Social Corporativa viene a estar integrada a lo largo de la entidad (empresa, ONG, cooperativa, corporación, administración pública), calando en cada práctica organizacional. Así, una empresa sostenible es un tipo nuevo y mejorado de corporación que acoge el desarrollo sostenible. En lugar de ver sus responsabilidades sociales como medios para obtener un objetivo ${ }^{26}$ entiende la sostenibilidad, y por tanto la RSC, como la razón de ser de la operación empresarial.

24 Subirats i Humet, J. (2005).

25 http://eur-lex.europa.eu/legal-content/ES/TXT/?uri=CELEX\%3A52001DC0366.

26 Por ejemplo, para obtener una licencia pública para operar o mejora de la imagen social de la empresa. 
Los negocios inclusivos (NI) son un ejemplo de esa intervención en el ámbito de lo económico, lo laboral y lo relacional. Son iniciativas empresariales económica, social y ambientalmente responsables, son negocios sostenibles que según la lógica del beneficio mutuo procuran mejorar las condiciones de vida de las comunidades empobrecidas incorporándolas en sus cadenas de valor para mejorar su calidad de vida. Un Negocio Inclusivo busca construir puentes entre el negocio y las poblaciones de bajos ingresos para el beneficio de ambos ${ }^{27}$.

La definición del término "Negocio Inclusivo" varía de acuerdo con la entidad que lo promueve; en términos generales busca incluir a las personas que pertenecen a la base de la pirámide dentro de su cadena de valor, ya sea como consumidores, proveedores, distribuidores o clientes, para que ellos puedan tener acceso a bienes, servicios y una mejor calidad de vida ${ }^{28}$.

Establecen una relación entre empresa y persona con bajos ingresos, que se constituyen como socios empresariales, proveedores/distribuidores y/o consumidores pudiendo poner en el mercado productos y servicios que cubren sus necesidades en unas condiciones de accesibilidad económica. Se caracterizan por ser sostenibles a nivel económico, de progreso social y con un balance ambiental positivo.

Si bien el art. 5 de la Ley 5/2011 de economía social enumera una serie de entidades que conformarían este sector, esta enumeración no es cerrada sino que queda abierta a otras formas jurídicas que persigan los principios de la economía social de satisfacer las necesidades sociales. Los NI pueden acogerse a cualquiera de las figuras contempladas (como son las cooperativas, las asociaciones y fundaciones o las sociedades agrarias de transformación, entre otras) pero no es necesario que sea así para que se puedan considerar inclusivos. Para que un negocio sea considerado como inclusivo tiene que tener una filosofía de empresa propia que se puede plasmar en cualquier forma empresarial, también en una sociedad limitada o una sociedad anónima, siempre que cumpla con las características de implicar a las BdP.

La relación entre Responsabilidad Social Corporativa y NI es clara pues conlleva una comprensión más holística y completa de los stakeholder ${ }^{29}$ al

27 CECODES (2008).

28 CECODES (2010); The World Bank (2016).

29 Grupos de interés de una empresa, a quienes pueden afectar o son afectados por las actividades de la misma. 
darles, no sólo voz a la hora de diseñar la gestión del negocio empresarial y ser posibles receptores de la acción social empresarial, sino que se les integra a lo largo de la empresa y de su cadena de valor, respondiendo plenamente las cuestiones sociales, mejorando las posibilidades económicas de los consumidores, distribuidores y proveedores, aumentando los consumidores potenciales y todo ello desde una perspectiva de sostenibilidad ambiental.

Su aplicación implica la construcción de una relación de confianza entre las partes, a partir de una colaboración activa y equitativa ${ }^{30}$. Los actores que figuran (Figura 3) en estos negocios tienen la misma importancia y se necesitan mutuamente: las empresas generan oportunidades de negocio y ofrecen conocimiento del mercado; el tercer sector (fundaciones, asociaciones y cooperativas así como empresas de economía social) tienen la experiencia en el trabajo de campo, con las comunidades y, por tanto, su conocimiento; el estado puede ofrecer acceso a financiación, regular una legislación facilitadora y tiene acceso a las poblaciones interesadas; la universidad, ofrece modelos y conocimiento así como la posibilidad de realizar investigación al respecto; y la comunidad participaría en la cadena de valor de las empresas pudiendo ofrecer/recibir servicios y bienes de éstas.

\section{Figura 3}

\section{Los negocios inclusivos y sus actores}

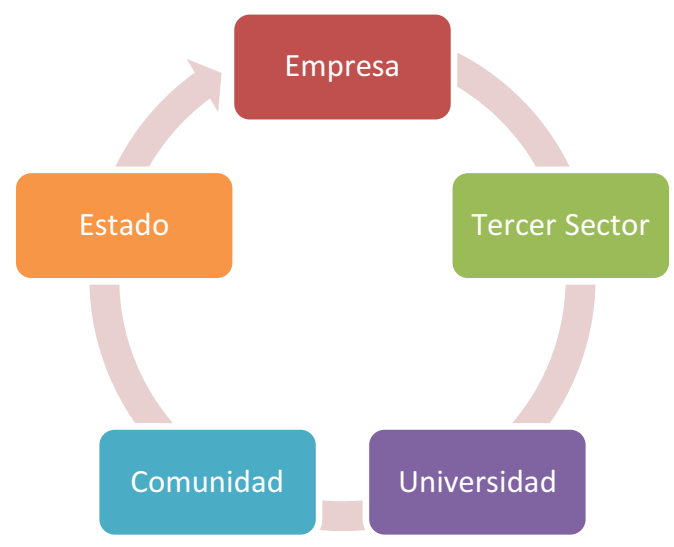

Fuente: Elaboración propia.

$30 \mathrm{http} / / /$ www.cecodes.org.co/negocios.html 
Estas iniciativas pueden contribuir a la reducción de la pobreza, y al tiempo generar mayor acceso a oportunidades facilitando que las comunidades en las que se desarrollan sean, a su vez, generadoras de ingresos y puedan ejercer el derecho a la libertad económica. Es decir, a estas comunidades se les dota de mayor eficiencia, sostenibilidad e innovación generando también oportunidades para el sector privado.

En los países desarrollados se trabajan los Negocios Inclusivos, principalmente desde la economía social; desde la empresa social entendida como acción de emprendimiento social y económico, cuyo modelo de empresa acentúa sus intereses en la participación democrática de sus actores y la búsqueda de la mejora de colectivos socialmente desfavorecidos, y que pretende generar un cambio en el modelo socioeconómico.

Este modelo de empresas permite acercar servicios y crear empresas con valor social para colectivos tradicionalmente excluidos como nicho de mercado al ser vistos como meros beneficiarios de los sistemas públicos de servicios sociales ${ }^{31}$.

La empresa murciana Neosistec es un ejemplo de éxito español de este modelo de trabajo en el que una empresa tecnológica, con apoyo de fondos económicos públicos y privados, y en colaboración con investigadores de la universidad y de ONGs del entorno especializadas en el trabajo con personas con discapacidad visual, crea una serie de aplicaciones para el móvil, de descarga gratuita, que genera un aumento de la calidad de vida de las personas con problemas de visión a través de las aplicaciones para el móvil "Supervisión", "Visual Tags" y sus nuevo sistema galardonado "Navilens".

El concepto de la "Base de la Pirámide" (BdP), se plasma gráficamente en una pirámide que contiene en la cúspide a las personas con mayores ingresos y capacidad adquisitiva, y en la parte inferior se encuentran las personas que viven con un ingreso diario igual o menor a US\$2.00. Prahalad y Hart ${ }^{32}$ fueron los creadores de este concepto, y propusieron que las grandes multinacionales deberían aprovechar los mercados emergentes, al encontrarse estos en proceso de desarrollo en diferentes países con un alto porcentaje de personas que pertenecían a la base piramidal. Planteaban a las empresas reevaluar el valor/rendimiento de sus productos y servicios para que fueran ofrecidos a las personas que pertenecían con bajos ingresos, lo que a su vez supondría una

31 Sánchez, P. (2012). 
mejora en su calidad de vida, convirtiéndolos en productores o distribuidores de los productos que fueran ambientalmente sostenibles y económicamente viables ${ }^{33}$.

Este protocolo de desarrollo empresarial con Base de la Pirámide nos permite a los profesionales del ámbito de lo social involucrar a las empresas en la lucha contra la pobreza y la exclusión desde una intervención comunitaria, ya que tiene como objeto principal crear "valor mutuo" mediante la "cocreación"; generando valor mutuo no solo se consigue crear un nuevo negocio, sino que cada etapa del proceso genera valor para todos los socios. Por otro lado, el término co-creación busca trabajar en igualdad de condiciones con las comunidades para imaginar, desarrollar y alcanzar un negocio sostenible que sea de todos los participantes ${ }^{34}$.

Otra forma de luchar contra la exclusión social es contar con empleados en situación de riesgo, creando una red de trabajo a través de convenios con empresas de inserción de la zona, lo que permitiría compartir recursos. Las empresas de inserción son, también, entidades de iniciativa social que, como establece el art. 1 de la Ley 44/2007 para la regulación del régimen de las empresas de inserción, tienen por objeto promover la inserción sociolaboral de personas en situación de exclusión social. Explica Askunze (2016) que no se consideran una nueva modalidad de modelo societario sino que pueden adoptar cualquier forma jurídica, si bien debe estar legalmente constituida por una entidad sin ánimo de lucro como patrocinadora, y ésta debe contar al menos con el $51 \%$ del capital social de la sociedad mercantil.

Esta metodología plantea realizar un trabajo conjunto con la empresa y con las personas que pertenecen a la comunidad en la cual se está desarrollando el proyecto. Se genera la inclusión social mediante acciones focalizadas en la incorporación de comunidades y familias sin recursos económicos en la economía de mercado a través del abordaje socio-cultural, complementario del económico. Se plantea un trabajo con las diferentes comunidades para lograr desarrollar negocios que sean sostenibles en el tiempo y mejoren su calidad de vida. De esta manera, reconociendo la pobreza como un fenómeno multidimensional determinado no solamente por factores económicos sino

32 Prahalad, C. K. y Hart, S. (2002).

33 Prahalad, C. K. y Hart, S. (1999); Gardetti, M. A. (2009).

34 London, T.; Anupindi, R. y Sheth, S. (2010); London, T. y Hart, S. L. (2004); Simanis, E. y Hart, S. L. (2008) en Ishikawa Lariú, A. y Strandberg, L. (2009), p. 18. 
también por fuerzas políticas y sociales que inhiben el desarrollo humano y el potencial empresarial ${ }^{35}$, los estudiosos de la Base de la Pirámide proponen una aproximación diferente al problema de la pobreza en estas sociedades, acercándose al mismo desde una nueva perspectiva de mercado.

Ejemplo de este modelo de trabajo en España lo encontramos en la iniciativa del BSCH, con la puesta en marcha de International Express, destinado a ofrecer servicios financieros a trabajadores extracomunitarios. Para diseñar el funcionamiento de estos servicios se realizó un estudio de campo para conocer las necesidades de los futuros clientes; estableció alianzas con otras entidades bancarias en los países extracomunitarios, fijando comisiones mínimas e iguales para todos los países y con todos los bancos corresponsales, lo que permitió garantizar la efectividad del servicio, la transparencia del precio y seguridad en los envíos. Todo esto unido a una estrecha relación con ONGs e instituciones vinculadas con la población inmigrante permitió también fortalecer la imagen de seguridad y de confianza en la marca BSCH e International Express.

Las premisas que rigieron este modelo fueron, al igual que para cualquier negocio con BdP, en un primer paso ofrecer confianza al cliente al atender las demandas identificadas en el estudio de campo, ofrecer servicios y productos personalizados que daban respuesta a las necesidades del colectivo inmigrante, y tener unos empleados formados y sensibilizados con estos clientes, lo que permitió captarlos como nuevos clientes y, posteriormente, ampliar la cartera de servicios vinculados. $\mathrm{Al}$ mismo tiempo esto permitió mejorar la reputación de la entidad bancaria entre los trabajadores extracomunitarios ${ }^{36}$.

\section{EL PROFESIONAL DE LO SOCIAL COMO AGENTE DE RSC}

\section{Trabajo social y Derechos Humanos}

La definición de trabajo social aportada por la Federación Internacional de Trabajadores Sociales (FITS) y la Asociación Internacional de Escuelas de Trabajo Social (IASSW) manifiesta que "La profesión de trabajo social promueve el cambio social, la resolución de problemas en las relaciones

35 Tashman, P. y Marano, V. (2010).

36 Iesse.edu/en/files/ciilejemplos_tcm4-5531.pdf. 
humanas, y el fortalecimiento y la liberación del pueblo, para incrementar el bienestar. Mediante la utilización de teorías sobre el comportamiento humano y los sistemas sociales, el trabajo social interviene en los puntos en los que las personas interactúan con su entorno. Los principios de los Derechos Humanos y la Justicia Social son fundamentales para el Trabajo Social". Y es que el vínculo entre Trabajo Social y Derechos Humanos existe desde los inicios de nuestra profesión.

En 1995 el Centre for Human Rights de la ONU, en conjunto con la FITS y IASSW, publica por primera vez un manual para escuelas de servicios sociales donde se considera imperativo "que las personas que participan en la enseñanza y la práctica del servicio social se comprometan claramente, sin reservas, en la promoción y en la satisfacción de las aspiraciones sociales fundamentales"

Muestra del vínculo entre Derechos Humanos y Trabajo Social es el listado de los siete instrumentos de Derechos Humanos considerados de esencial relevancia por la FITS para la práctica y acción del trabajador social en materia de empresa ${ }^{37}$, entre los que se encuentran la Carta de las Naciones Unidas (1945) $)^{38}$, Declaración Universal De Derechos Humanos (1948) ${ }^{39}$, Convención Internacional sobre la eliminación de todas las formas de discriminación racial $(1965)^{40}$, los Pactos Internacionales de Derechos Humanos (Pacto Internacional de Derechos Civiles y Políticos ${ }^{41}$ y Pacto Internacional de Derechos Económicos, Sociales y Culturales (1966) ${ }^{42}$, Convención sobre la eliminación de todas las formas de discriminación contra la mujer (1979) ${ }^{43}$, Convención contra la tortura y otros tratos o penas crueles, inhumanos o degradantes $(1984)^{44}$, Convención sobre los Derechos del niño $(1989)^{45}$, Convención Internacional sobre la protección de los derechos de todos los trabajadores migratorios y de sus familiares $(1990)^{46}$, así como convenios de la OIT tales como Trabajo forzoso ${ }^{47}$, aprobado en 1930, Igualdad

37 Naciones Unidas, (1995).

38 http://www.un.org/es/charter-united-nations/.

39 http://www.un.org/es/documents/udhr/.

40 http://www.ohchr.org/Documents/HRBodies/CERD/ICERDManual_sp.pdf.

$41 \mathrm{http} / / /$ www.ohchr.org/SP/ProfessionalInterest/Pages/CCPR.aspx.

42 http://www.un.org/es/events/culturaldiversityday/covenant.shtml.

43 http://www.un.org/womenwatch/daw/cedaw/text/sconvention.htm.

$44 \mathrm{http} / / /$ www.ohchr.org/SP/ProfessionalInterest/Pages/CAT.aspx.

$45 \mathrm{http} / / /$ www.ohchr.org/SP/ProfessionalInterest/Pages/CRC.aspx.

46 http://www.ohchr.org/SP/ProfessionalInterest/Pages/CMW.aspx.

47 http://www.ilo.org/dyn/normlex/es/f?p=NORMLEXPUB:12100:0::NO::P12100_ILO_CODE:C029. 
de remuneración $(1951)^{48}$, Edad mínima de admisión al empleo $(1973)^{49}$, Trabajadores migrantes $(1975)^{50}$ y Trabajadores con responsabilidades familiares $(1981)^{51}$.

\section{El trabajador social y la empresa}

Los trabajadores sociales en el ámbito empresarial pueden influir en el diseño y construcción de los planes de Responsabilidad Social Corporativa, pues esta surge del estudio y debate del impacto que la empresa tiene con su actuación, sus contribuciones y las consecuencias de las prácticas comerciales en los Derechos Humanos y en las personas. La Responsabilidad Social Corporativa es el compromiso voluntario que una entidad contrae para que su gestión corporativa sea una gestión social, ya que atiende al impacto que su actividad tiene a nivel económico, social y medioambiental, juzgando que con una actividad sostenible y respetuosa, a la vez que económicamente rentable, puede beneficiarse la propia corporación, la comunidad en la que se encuentra enmarcada y el resto de actores con los que se relaciona.

Si se entiende la Responsabilidad Social Corporativa desde un enfoque de aplicabilidad y justiciabilidad basada en los derechos socioeconómicos, aparece como un "desarrollo realizado por el sector privado y [perfectamente complementado] de los esfuerzos de desarrollo de los gobiernos y las instituciones multilaterales de desarrollo" ${ }^{52}$.

La Responsabilidad Social Corporativa supone la búsqueda de una nueva forma de gestión empresarial, inspirada en la ética y los principios de los Derechos Humanos, en una sociedad en la que la sociedad ve una mayor desigualdad social global. Las empresas no pueden quedarse al margen de los problemas y retos a los que se enfrenta la sociedad, y que la razón económica no puede justificar unas desigualdades hirientes y la exclusión social de grupos de personas cada vez más amplios en todos los países desarrollados" ${ }^{53}$.

El trabajador social puede inspirar una nueva praxis en el ámbito de la empresa, ya que la Responsabilidad Social tiene dos dimensiones de actuación,

\footnotetext{
48 http://www.ilo.org/dyn/normlex/es/f?p=1000:12100:0::NO::P12100_ILO_CODE:C100.

49 https://www.ilo.org/legacy/spanish/buenos-aires/trabajo.infantil/resource/docs/sabermas/normativa/c138.pdf.

50 http://www.ilo.org/dyn/normlex/es/f?p=1000:12100:0::NO::P12100_INSTRUMENT_ID:312288.

51 http://www.ilo.org/dyn/normlex/es/f?p=NORMLEXPUB:12100:0::NO::P12100_ILO_CODE:C156.

52 Vives, A: (2004), p. 46 en Osuji, O. K. (2014), p. 14.

53 Perdiguero, (2003), pp. 17-18 en Carrasco Arnal, J. (2009), p. 74.
} 
una interna referida a las propias prácticas que la empresa puede desarrollar dentro de sí misma (fomento de una legislación equitativa en cuanto al género, fortaleciendo las relaciones de las minorías raciales con la mayoría de los trabajadores y con otras minorías, diagnosticando y promoviendo cambios para evitar los conflictos que la religión puedan tener en el ejercicio de la profesión, etc.), y otra dimensión externa, enfocada al desarrollo social de las comunidades donde operan (participación y el compromiso de las comunidades; la transferencia de tecnología; el respeto de los conocimientos, los métodos y las prácticas autóctonas y locales, entre otros).

El trabajo social de empresa quedó definido por Bernard (1967) como aquella "actividad organizada, que pretende ayudar a la adaptación recíproca de los trabajadores y su empresa. Este objetivo se alcanza mediante la utilización de técnicas y métodos destinados a permitir que los trabajadores, los grupos y las colectividades de trabajo, hagan frente a las necesidades, resuelvan los problemas que plantea su adaptación a una sociedad industrial en evolución y, merced a una acción cooperativa, que mejoren las condiciones económicas y sociales". Para este autor, nos recuerda Raya Díaz "el papel del trabajador social en la empresa se articula en torno a tres planos: 1. Dar apoyo psicosocial a los individuos y colectividades de trabajadores que se encuentran en dificultades; 2. Participar en la creación, funcionamiento y mejora de las realizaciones sociales de la empresa; y, 3. Favorecer una mejor toma de conciencia de los problemas humanos y sociales surgidos con motivo del trabajo" ${ }^{54}$. Es decir, darían respuesta a esa dimensión interna.

De estas dos dimensiones, de la Responsabilidad Social Corporativa y de la concepción del Trabajo Social, se desprenden dos perfiles profesionales para el trabajador social en la empresa: el trabajador social empresarial para la dimensión externa, y el trabajador social de empresa, más orientado a la dimensión interna.

El trabajador social empresarial tiene su ámbito de intervención más allá de la organización interna de la empresa e incluye a los stakebolders: clientes, proveedores, socios comerciales, consumidores, autoridades públicas y organizaciones sin ánimo de lucro. La responsabilidad se demuestra fundamentalmente en la gestión del negocio y en la forma en la que la empresa "puede colaborar con la sociedad reinvirtiendo en ella o compartiendo con

54 Raya Díaz, E. y Caparrós Civera, N. (2013), p. 341. 
ella los activos conseguidos. A esta forma de responsabilidad se le llama acción social de la empresa" ${ }^{55}$.

Desde esta perspectiva, Morros y Vidal $^{56}$ definen acción social empresarial como "el conjunto de acciones no lucrativas de la empresa, cuya misión es contribuir a mejorar la calidad de vida de las comunidades donde actúa, mediante proyectos sociales, formativos, culturales o medioambientales". Valor Martí (2013) define la acción social como la estrategia sostenida de inversión en la comunidad, que trata de alinear los objetivos empresariales con las necesidades sociales, medio ambientales y económicas de la comunidad en la que opera la empresa, con el fin de promover los intereses a largo plazo de la empresa y reforzar su reputación, al tiempo que se resuelven de forma efectiva las necesidades de la comunidad.

A nivel interno, el trabajador social de empresa, en materia de RSC, podría ser responsable tres áreas, de la gestión de los recursos humanos, de la salud y seguridad en el trabajo, y de las relaciones sociales que dentro de la empresa se producen. Desde una dimensión externa el trabajador social empresarial será el responsable también de una cuarta área que sería la de la Responsabilidad Social Corporativa externa. Y casi en los mismos términos Raya y Caparrós ${ }^{57}$ recogen las áreas de esta figura profesional: Salud en el Trabajo, Relaciones Laborales, atención a situaciones personales y familiares de los empleados y proyección social de la empresa y la Responsabilidad Social Corporativa.

A continuación se analizan detalladamente cada una de las áreas de trabajo y se describen las tareas concretas que podrían comprender, conforme se aprecia en la Figura 4.

\footnotetext{
55 Valor Martí, C. (2013), p. 56.

56 Morros, J. y Vidal, I. (2005), pp. 75-76.

57 Raya Díaz, E. y Caparrós Civera, N. (2013), p. 344.
} 


\section{Figura 4}

Áreas Responsabilidad Social Corporativa y actividades a desarrollar

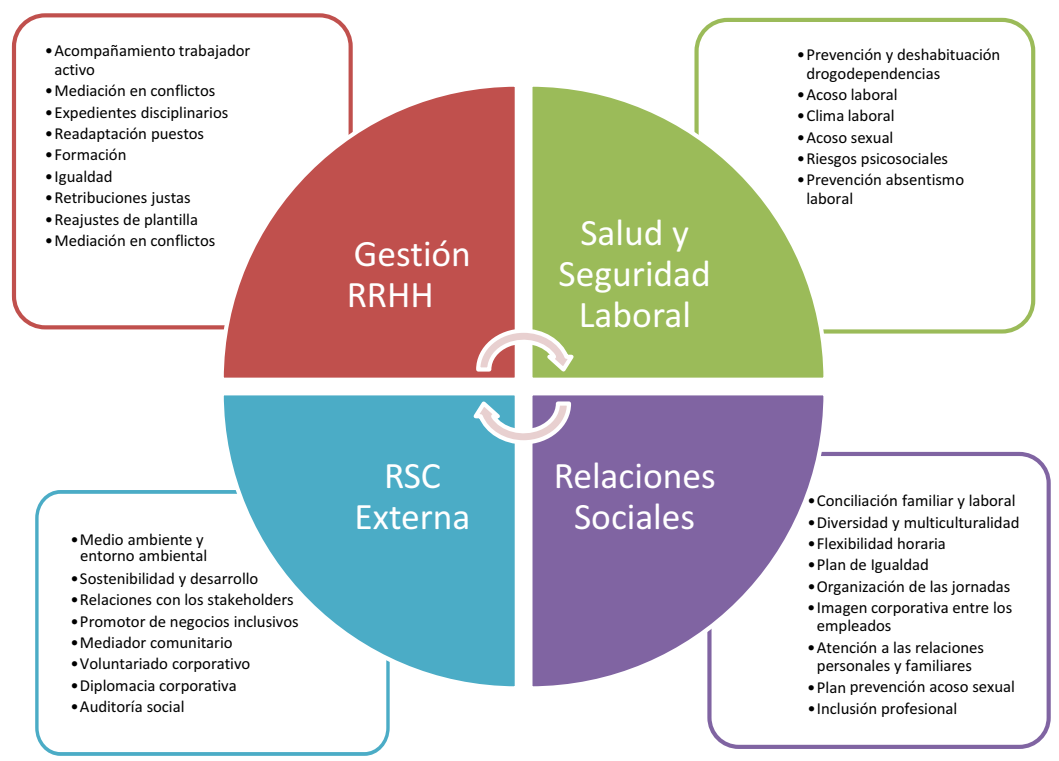

Fuente: Elaboración propia

El trabajador social empresarial realizará las tres primeras áreas, a saber, 1) gestión de recursos humanos, 2) salud y seguridad en el trabajo y 3) relaciones sociales.

La gestión de los recursos humanos supone estar al servicio de las personas contratadas durante todo su tiempo en activo en la empresa, desde la acogida de un nuevo miembro en la plantilla hasta el momento de su cese por jubilación, incapacidad o despido, pasando por la gestión de las vacaciones, los permisos o los periodos de incapacidad, las retribuciones justas de los trabajadores, la conciliación laboral, la adaptación del puesto de trabajo a las necesidades y características específicas del empleado, las perspectivas profesionales, reajustes de la plantilla (procesos de incorporación o de ERE), mediación en conflictos, asesoramiento en expedientes disciplinarios, de readaptación del puesto o de ascensos, y detección de formación del personal. 
La salud y seguridad en el trabajo conllevarían el estudio, seguimiento, tratamiento y evaluación de los riesgos psicosociales, como son la prevención y deshabituación de drogodependientes, gestión del estrés y del acoso laboral, prevención del absentismo laboral y análisis del clima laboral. La detección, medición, impacto, evaluación y prevención para la gestión de la salud y seguridad laboral implica llevar un registro de los accidentes de trabajo, enfermedades profesionales y los incidentes ocupacionales para poder analizar si hay algún factor humano que se pueda modificar para evitar o al menos reducirlos, a través de registros y estadísticas, pero también de entrevistas personales con el personal, lo que nos facilitará información sobre cómo ha ocurrido, cuáles son las consecuencias para el trabajador, el resto de los compañeros y la empresa o qué se podía haber hecho para evitarlo

En materia de relaciones sociales, y estas son las cuestiones para las cuales mejor preparados se encuentran los trabajadores sociales, encontramos: la conciliación familiar y laboral, atendiendo en la medida de lo posible a la flexibilidad horaria y tipos de jornada; atención a la diversidad y a la interculturalidad, trabajando desde el modelo de concepción del trabajador como persona con derechos sociales, económicos, políticos, ecológicos, laborales y culturales; del diseño, implementación, seguimiento y evaluación de los planes de igualdad (dentro de los cuales se deben volver a recoger los temas de conciliación, interculturalidad, además de incluir la perspectiva de género), resolución de conflictos en el ámbito laboral; planes de acoso sexual (formación, detección, denuncia, intervención); inclusión de personas en riesgo de exclusión social, a través de formación del personal con talleres de sensibilización en esta materia y puestos de trabajo adaptados y/o reservados para personas en situación de exclusión o de riesgo social para facilitar su inclusión laboral (aquí se entiende desde integración de personas con discapacidades o de minorías étnicas, a mujeres maltratadas, personas con bajo nivel de ingresos o sin estudios, personas que vienen de situación de falta de hogar, o incluso, de personas que vienen de ser trabajador sexual).

A través de la Responsabilidad Social Corporativa externa la empresa pretende reducir el impacto negativo que sus actividades empresariales suponen para el entorno, el medio ambiente y las relaciones con la comunidad. Esta forma de intervención social en la empresa debe partir del uso de los instrumentos del trabajo social que permiten el estudio de una comunidad y su problemática, el diagnóstico social, el trabajo con grupos y comunidades, la elaboración del proyecto social, etc.: 
- Como agente de promoción y facilitación de la Responsabilidad Social Corporativa en la comunidad, donde realizaría las funciones de movilizador comunitario y de asesoramiento a la sociedad civil y ONGs sobre acciones sociales

- Respecto al medio ambiente y entorno, se desarrollan funciones de promoción del medio ambiente y la vida sana, organización, implementación y participación en actividades destinadas a compensar la actividad empresarial en el entorno urbano y el medio ambiente, promover y apoyar medidas encaminadas a la reducción del impacto ambiental y elaborar y difundir las buenas prácticas en este sentido.

- En inversión social: voluntariado corporativo; accesibilidad social; educación de colectivos desfavorecidos; integración social; política de inversión en la comunidad; desarrollo local y comunitario; acción social; derechos laborales y sociales; creación de capital social; necesidades sociales básicas; ciudadanía responsable; atención de personas con discapacidad; auditoria social; diplomacia corporativa (mediación institucional, económica y social...

- Cuidando las relaciones de la empresa con la comunidad, que incluye la relación con las administraciones públicas, con la comunidad en el que se desarrolla la actividad empresarial y el tercer sector.

Administraciones públicas, a través de, por ejemplo, la colaboración y asesoramiento en políticas sociales, el diseño de protocolos de coordinación para acciones conjuntas, el diseño de la acción social empresarial compaginada con los servicios sociales públicas, $\mathrm{o}$ análisis de las medidas políticas públicas en materia de empresa, empleo, inserción, juventud, discapacidad, etc., que puedan afectar a la empresa.

Comunidad y Tercer Sector: elaboración y evaluación de proyectos sociales: las subvenciones a proyectos sociales es una de las formas de intervención más identificables en una comunidad (de cooperación, becas de formación académica o laboral) junto con el mecenazgo de artistas, deportivas o culturales, y el voluntariado corporativo. 


\section{CONCLUSIONES}

La ONU proclamó la Declaración Universal de Derechos Humanos y reconoció el deber de los Estados de proteger los Derechos Humanos siendo uno de sus principios el de cooperación pacífica entre los Estados. Sin embargo, los estados no deben operar solos sino que deben marcar, mediante la legislación, cómo se defienden y establecer el papel del resto de los actores, sociedad civil y empresa.

La empresa debe convertirse, y de hecho se está convirtiendo, en un motor de cambio individual, grupal y comunitario. La globalización tiene sus luces y sus sombras, puede crear oportunidades y destruir beneficios, de ahí la importancia del Estado como legislador y supervisor de que se cumple con la normativa internacional y nacional en materia de DDHH.

La Justicia Social que proclaman las naciones se basa en cómo las instituciones sociales distribuyen deberes y derechos en la sociedad, y el hecho de que las empresas se unan en la construcción de esa Justicia las convierte en un tipo nuevo y mejorado de corporación que acoge el desarrollo sostenible. Dos ejemplos de estos tipos de entidad son los Negocios Inclusivos, que intervienen en el ámbito de lo económico, lo laboral y lo relacional, y las empresas con Base de Pirámide, que ofrecen sus productos y servicios a las personas que pertenecían con bajos ingresos.

Además, desde las grandes corporaciones internacionales hasta las PYMES están desarrollando iniciativas de RSC, las cuales para ser realmente eficaces deben ser sensibles al contexto en el que se desarrollan, basadas en el tipo de entidad desde la que surge la iniciativa y debe ser sostenible económica y temporalmente.

El trabajador social es un profesional que puede ayudar a las empresas, entidades y corporaciones a crear su cadena de valor y a trabajar desde la ética empresarial.

Dos son los roles que el trabajador social puede desempeñar en el mundo de la empresa, el de trabajador social empresarial, aquel que se encarga de las relaciones externas y hacia el exterior de la empresa, y el de trabajador social de empresa, cuya actividad profesional está dirigida a los trabajadores y al lugar de trabajo desde el que ejerce.

Los cambios que vive la sociedad serán los mismos que se vean en las empresas, por lo que ésta deberá estar preparada, ser flexible y tener capacidad para conocer, adaptar y asumir esos cambios. 
El trabajador social es un agente de cambio, es un profesional conocedor, investigador y actor de la sociedad preparado para trabajar desde la empresa en aceptar y adaptarse a esos cambios. Como ejemplos podemos encontrar, entre otros, estas dos realidades: la mayor asunción del hombre en el rol parental se traduce en aumento de solicitudes de conciliación horario o de reducción de jornadas de trabajo, algo hasta ahora mayoritario entre las mujeres por lo que era prácticamente inimaginable que fuera a ser solicitado también por los trabajadores del sexo masculino; la mayor presencia de profesionales con distintas capacidades y discapacidades requieren de adaptaciones de puestos de trabajo y también de entender la forma de "estar en el trabajo", dando cabida al teletrabajo o trabajar por objetivos en vez de por presencialidad en un puesto físico de la empresa. 


\section{BIBLIOGRAFIA}

Askunze Elizaga, Carlos (2016), "Empresas de Inserción en la economía social. Herramientas para la inclusión socio-laboral", Revista furídica de Economía Social y Corporativa, no 29, CIRIEC, Madrid, pp. 15-49.

Barkemeyer, Ralf (2011), "Corporate Perceptions of Sustainability Challenges in Developed and Developing Countries: Constituting a CSR Divide?, Social Responsibility Fournal, vol. 7, n 2, pp. 257-281.

Bengoetxea Alkorta Aitor y Millán Calenti, Rafael (2016), "La forma jurídica de las entidades de la economía social", Revista furídica de Economía Social y Corporativa, n 29, CIRIEC, Madrid, pp. 9-14.

Castel, Robert (2004), "Encuadre de la exclusión”, en Karsz, Saúl (coord.), La exclusión: bordeando sus fronteras. Definiciones y matices, Gedisa, Barcelona, pp. 55-86.

Comisión Mundial sobre la Dimensión Social de la Globalización (2004), Por una globalización justa: Crear oportunidades para todos, Informe de la Comisión Mundial sobre la Dimensión Social de la Globalización, Ginebra.

CECODES (2008), Negocios Inclusivos: Iniciativas empresariales rentables con impacto de desarrollo, SNV and WBCSD, Quito.

CECODES (2010), Negocios Inclusivos: Creando valor en América Latina, SNV and WBCSD, Quito.

EUMEDNET (2014), Migraciones, Causas y Consecuencias. X Congreso Internacional sobre Inmigración, Universidad de Málaga, Málaga.

Frynas, Jedrjez George (2005), “The False Developmental Promise of Corporate Social Responsibility: Evidence from Multinational Oil Companies”, International Affairs, vol. 81, n 3, pp. 581-598.

Frynas, Jedrjez George (2008), "Corporate Social Responsibility and International Development: Critical Assessment", Corporate Governance: An International Review, vol. 16, n 4, pp. 274-281. doi:10.1111/j.1467-8683.2008. 00691.x.

Gardetti, Miguel Ángel (2009), "Los Stakebolders Periféricos y la Innovación para la Base de la Pirámide: hacia la co-creación y la intimidad de negocios”, en Gardetti, Miguel Ángel (Comp.), Textos sobre la Base de la Pirámide. Hacia la Co-Creación de Valor y Desarrollo, Instituto de Estudios para la Sustentabilidad Corporativa, Buenos Aires, pp. 75-90. 
Ishikawa Lariú, Alice y Strandberg, Lena (2009), "Negocios inclusivos: creando valor para las empresas y para la población de bajos recursos", Cuadernos de la Cátedra "la Caixa" de Responsabilidad Social de la Empresa y Gobierno Corporativo, IESE Business School, Madrid.

Jamali, Dima J. (2010), "The CSR of MNC Subsidiaries in Developing Countries: Global, Local, Substantive or Diluted?", Fournal of Business Ethics, vol. 93, Suppl 2, pp. 181-200.

Kolstad, Ivar (2009), "Human Rights and Assigned Duties: Implications for Corporations", Human Rights Review, vol. 10, n 4, pp. 569-582.

Lodge, George y Wilson, Craig (2006), A Corporate Solution to Global Poverty. How Multinationals Can Help the Poor and Invigorate Their Own Legitimacy, Princeton University Press, Princeton.

London, Ted; Anupindi, Ravi y Sheth, Sateen (2010), "Creating Mutual Value: Lessons Learned From Ventures Serving Base of the Pyramid Producers", Fournal of Business Research, vol. 63, nº 6, pp. 582-594

London, Ted y Hart, Stuart L. (2004), "Reinventing Strategies for Emerging Markets: Beyond the Transnational Model", Fournal of International Business Studies, vol. 35, pp. 350-370.

Márquez Carrasco, Carmen (Ed.) (2014), España y la implantación de los Principios Rectores de las Naciones Unidas sobre empresas y Derechos Humanos: oportunidades y desafíos, Huygens Editorial, Barcelona.

Martin-Ortega, Olga (2013), "La diligencia debida de las empresas en materia de derechos humanos: un nuevo estándar para una nueva responsabilidad”, en Zamora Cabot, Francisco Javier; García Cívico, Jesús y Salés Pallarés, Lorena (Eds.), La responsabilidad de las empresas multinacionales por violaciones de los derechos bumanos, Cátedra de Derechos Humanos y Oficina del Defensor del Pueblo, Universidad de Alcalá, Madrid, pp. 167-192.

Moix, Manuel (1977), La fusticia Social, incógnita de nuestro tiempo, Servicio de Publicaciones. Ministerio de Trabajo, Madrid.

Moriña, Anabel (2007), La exclusión social: análisis y propuestas para su intervención, Fundación Alternativas, Madrid.

Morros, Jordi y Vidal, Isabel (2005), Responsabilidad Social Corporativa. Madrid. Fundación Confemetal. 
Muthuri, Judy N; Moon, Jeremy e Idemudia, Uwafiokun (2012), "Corporate Innovation and Sustainable Community Development in Developing Countries", Business \& Society, vol. 51, n 3, pp. 355-381.

Naciones Unidas (1987), Derechos Humanos: Preguntas y respuestas, Naciones Unidas, Nueva York.

Naciones Unidas (1995), Derechos Humanos y Trabajo Social. Manual para escuelas de servicio social y trabajadores sociales profesionales, Serie de capacitación profesional n ${ }^{\circ} 1$, Naciones Unidas, Nueva York y Ginebra .

Naciones Unidas (2008), Consejo de Derechos Humanos, octavo período de sesiones. Proteger, respetar y remediar: un marco para la empresa y los Derechos Humanos. Informe del Representante Especial del Secretario General sobre la cuestión de los Derechos Humanos y las empresas transnacionales y otras empresas comerciales, John Ruggie, A/HRC/8/5.

Naciones Unidas (2011), Principios sobre las empresas y los Derechos Humanos: La implementación de las Naciones Unidas para "proteger, respetar y remediar" marco, Informe del Representante Especial del Secretario General sobre Derechos Humanos, John Ruggie, sobre la cuestión de los Derechos Humanos y las empresas transnacionales y otras empresas comerciales. Documento de la ONU A/HRC/17/de 31.

Noland, James y Phillips, Robert (2010), "Stakeholder Engagement, Discourse Ethics and Strategic Management”, International fournal of Management Reviews, vol. 12, $\mathrm{n}^{\circ}$ 1, pp. 39-49.

O’Neill, Onora (2001), “Agents of Justice”, Metaphilosophy, vol. 32, ${ }^{\circ}$ 1/2, pp. 180-195.

Osuji, Onyeka K. (2014 online 2016), "Rights and Corporate Social Responsibility: Competing or Complementary Approaches to Poverty Reduction and Socioeconomic Rights?”, Fournal of Business Ethics, vol. 136, ${ }^{\circ}$ 2, pp. 329-347.

Prahalad, C. K. y Hart, Stuart (1999), Strategies for the Bottom of the Pyramid: Creating Sustainable Development, Working Paper.

Prahalad, C. K. y Hart, Stuart [1998 (2002)], "The Fortune at the Bottom of the Pyramide", Strategy + Business, vol. 26, pp. 1-14.

Rawls, John (1995), Teoría de la fusticia Social, Harvard University Press, Cambridge. 
Raya Díaz, Esther y Caparrós Civera, Neus (2013), “Trabajo social en las relaciones laborales y la empresa: vías para el emprendimiento", Documentos de Trabajo Social, no 52, pp. 338-356.

Sánchez, Pablo (2012), Los negocios inclusivos en España. Una visión participativa y colaborativa de la empresa, Documento de trabajo $\mathrm{n}^{\circ} 14$ de la Cátedra Mango de RSC de la Escola Superior de Comerç Internacional Universitat Pompeu Fabra.

Simanis, Erik \& Hart, Stuart L. (2008), The Base of the Pyramid Protocol:Toward Next Generation BoP Strategy, Cornell University, Ithaca, Nueva York.

Subirats i Humet, Joan (Dir.) (2005), "Análisis de los factores de exclusión social”, Documentos de Trabajo de la Fundación BBVA, n 4, Institut de Govern I Polítiques Públiques (UAB), Barcelona, pp. 35-48.

Tashman, Pete y Marano, Valentina (2009), "Dynamic Capabilities and Base of the Pyramid Business Strategies", Fournal of Business Ethics, vol. 89, Supp. 4, pp. 495-514.

Tezanos, José Félix (Ed.) (2008), "Exclusión social, democracia y ciudadanía económica: la libertad de los iguales", Revista del Ministerio de Trabajo e Inmigración, $\mathrm{n}^{\circ}$ 75, pp. 17-30.

Valor Martí, Carmen (2013), "La contribución de la empresa con la sociedad: tipos y valoración de las formas de colaboración", Cuadernos de Trabajo Social, vol. 26, n 1, pp. 55-63.

Van Sandt, Craig W. y Sud, Mukesh (2012), "Poverty Alleviation through Partnerships: a Road Less Travelled for Business, Governments and Entrepreneurs", Fournal of Business Ethics, vol. 110, n 3, pp. 321-332.

Vives, Antonio (2004), "The Role of Multilateral Development Institutions in Fostering Corporate Social Responsibility", Development, vol. 47, $n^{\circ} 3$, pp. $45-52$.

World Bank (2016), Annual Report 2015, World Bank, Washington. 